\title{
Die Rechtfertigung der Gier und die Finanzkrise
}

\author{
Justification of Greed and the Financial Crisis
}

\begin{abstract}
The aim of this article is at first to answer the question of greed (the justification of this "virtue" in the modern economic system), which is meant, in the light of Christian social ethics to be the primary cause of the present economic crisis. In the three steps of catholic social teaching (see, judge, act) we want to interpret the signs of our time (present age problems) in the light of the Gospel (and the teaching and tradition of the Church). The relevant question we have to pose in our statement is: Why does catholic social teaching, in opposite to the modern view of economic and political science, object to a justification of greed? The third (practical) step of our explanations we can paraphrase with the question: what shall we do? The focusing on institutional reforms, the belief in arrangements which exclusively accentuate sociological key aspects and believe in changes of structures (in education, politics, economics and welfare) led not only to market crisis, but also to a crisis of culture. If the human being in his/her deepest dimension (the ecology of person) is suffering, then the Church cannot remain silent, because its mission and way is the person. Not until we - like king Solomon - turn to God and ask for help and the ability to judge between good and evil, just and unjust, shall we find a safe basis to lead us out of despair and disorientation into a more humane civilization of humanity and solidarity.
\end{abstract}

\section{Keywords}

A justification of greed, the ecology of person, economic crisis, catholic social teaching.

Ziel dieses Artikels ist, wie im Titel angedeutet, die Beantwortung der Frage: Warum die Rechtfertigung der Gier zur Wirtschaftskrise führte. Dass wir eine Krise der Banken (durch Spekulanten verursacht) haben, brauchen wir nicht zu beweisen. Es ist eine Tatsache, die jeder täglich hören und erfahren 
kann. Die philosophische Frage, die dahinter steht, ist nicht beantwortet mit einer Analyse der ökonomischen oder politischen Gründe, die dazu geführt haben, dass wir von Ausschreitungen in Griechenland oder Spanien Berichte erhalten und uns Sorgen machen, ob der Euro und die Europäische Union als wirtschaftspolitisches Modell überhaupt überleben werden, sondern sie ist eher kultureller Natur, die nach den Ursachen dafür sucht, warum die Gier gerechtfertigt wurde. Diese Frage ist sehr eng mit einer Kapitalismuskritik verbunden, oder im Dreischritt der katholischen Soziallehre, mit der Beschreibung der gegenwärtigen Zeichen der Zeit (Sehen) ${ }^{1}$. Die zweite Frage, die wir in unseren Ausführungen stellen müssen lautet: Warum lehnt die katholische Soziallehre die Rechtfertigung der Gier ab? Das würde dem zweiten Schritt (Urteilen) entsprechen, einer Analyse und Interpretation im Lichte des Evangeliums (und der Tradition und Lehre der Kirche). Der dritte Schritt (Handeln) lässt sich mit der Frage umschreiben: Gibt es Auswege aus der Quadratur des Kreises, oder was sollen wir tun?

\section{Warum wird die Gier gerechtfertigt?}

Obwohl Adam Smith (1723-1790), der Urgroßvater der kapitalistischen Wirtschaftstheorie als ein Moralphilosoph ${ }^{2}$ in seinem berühmten Werk ,der Wohlstand der Nationen" nicht nur über das Eigeninteresse als Motor des wirtschaftlichen Handelns geschrieben hat, sondern auch Solidarität und moralische Werte vorausgesetzt hat ${ }^{3}$, wurde offiziell durch die Interpreten angenommen, dass in wirtschaftlichen Bereichen nur das egoistische Eigeninteresse und die liberale Freiheit (freie Hand der Märkte) die

1 Der Terminus Zeichen der Zeit, stammt aus der Enzyklika „Pacem in Terris“ Johannes XXIII., der die Aufgabe der christlichen Sozialethik anknüpfend an die Forderung Jesu, die Zeichen der Zeit zu erkennen, als die Hauptaufgabe und als Einladung zu verantwortungsbewusstem Handeln an jeden Christen richtet. W. Kirchschläger schreibt dazu: „Johannes XXIII. hat der Kirche damit das Grundmodell und die Methode für ihr eigenes Leben in Erinnerung gerufen und zugleich angedeutet, wie dies zu verwirklichen ist: Nicht, indem auf die Unglückspropheten gehört wird, die aus einer nach rückwärtsgewandten Perspektive die Gegenwart schlecht machen, sondern indem im Vertrauen auf das Wirken des Geistes die Kirche sich anschickt, ,einen Sprung nach vorwärts“ zu wagen, um ,in der Welt von heute“ die christliche Botschaft zu verkündigen“. www.unilu.ch/ files/die-zeichen-der-zeit-erkennen.pdf (30.08.2012).

2 Er hat auch eine Theorie der ethischen Gefühle geschrieben.

3 A. Smith, Der Wohlstand der Nationen, München 2001, S. 371, 124n, 407nn.; Z. Waleszczuk, Globalizacja solidarnosci, Wroclaw 2007, S. 69. 
ausreichenden Voraussetzungen einer gut funktionierenden Wirtschaft seien ${ }^{4}$. „Das Mittel, um neue Finanzprodukte attraktiv zu machen war denkbar einfach: Die menschliche Gier"5. Damit wurde die Wirtschaft von der Ethik getrennt, oder anders gesagt, der Egoismus hoch gelobt und gerechtfertigt ${ }^{6}$. Im „Wohlstand der Nationen“ lesen wir: „Nicht vom Wohlwollen des Metzgers, Brauers und Bäckers erwarten wir unsere Mahlzeit, sondern von deren Bedachtnahme auf ihr eigenes Interesse. Wir wenden uns nicht an ihre Menschenliebe, sondern an ihre Eigenliebe und sprechen ihnen nie von unseren eigenen Bedürfnissen, sondern von ihren Vorteilen" ${ }^{67}$. Der Ausgangspunkt der Theorie Adam Smiths trug dabei metaphysische Züge, der schottische Philosoph ging davon aus, dass der nach eigenem Interesse handelnde Unternehmer sich trotzdem (mit Selbstverständlichkeit) an die moralischen Normen hält. Anders gesagt, dass er in seiner Profitsuche andere Menschen (Konsumenten) wie seine Mitbrüder (Personen), behandelt und nicht mit allen machbaren, technisch möglichen Mitteln austrickst und ausnutzt (instrumentalisiert), um die Größe seiner Profite zu vermehren.

Leider ist es aber dazu gekommen, dass man die moralische Seite von Smiths Theorie immer häufiger als zweitrangig und irrelevant betrachtete, bis hin zu der Meinung, dass die Ökonomie (Wirtschaftslehre) gar keine Ethik und moralischen Voraussetzungen benötigt, um wirksam zu werden ${ }^{8}$. Es kam zur Herausbildung des Begriffs ,homo öconomicus ${ }^{69}$. Über dieses Problem gibt es viele interessante und ausführliche Abhandlungen, an dieser Stelle wollen wir jedoch lediglich betonen, dass die sogenannte Ökonomisierung des gesellschaftlichen (kulturellen) Lebens ein Faktum

${ }^{4}$ „Seiner Logik muss sich eine Politik, die erfolgreich sein will, unterwerfen. Immer mehr Lebensbereiche, die eine gewisse Autonomie gegenüber wirtschaftlichen Einflüssen bewahren konnten, werden ökonomisiert und damit der kapitalistischen Logik unterworfen“. P. Schönfelder, Pax-Christi-Kommission Weltwirtschaft (Hg.), Der Gott Kapital. Einführung, Berlin 2006, S. 18.

5 B. Gorissen, Glaube statt Inflation, Die Tagespost Nr. 142 (27.11.2012), S. 9.

6 „Besonders die Rolle der Banken ist hierbei kritisch zu sehen. Sie sammelten große Mengen an Kapital an, ohne substanzielle Werte für die Gesellschaft zu schaffen (...)“. Ebenda. Der Turbokapitalismus zielt auf die egoistische Bereicherung ab.

7 A. Smith, Der Wohlstand der Nationen, München 2001, S. 98,99. Vgl. R. Marx, Das Kapital, München 2008, S. 73.

8 N.O. Oermann, Anständig Geld verdienen?, München 2007, S. 20-21, S. 31.

9 A. Diez, Der homo oeconomicus in der Perspektive theologischer Wirtschaftsethik, Heidelberg 2004, S. 30. 
geworden ist ${ }^{10}$. Dies bedeutet, dass diese im Kern materialistische Sicht der Wirklichkeit, die das egoistische Eigeninteresse rechtfertigt, die Oberhand gewann und nicht nur im Bereich der Wirtschaft Anwendung fand, sondern $\mathrm{zu}$ einem Menschenbildprogramm erhoben wurde, was auch die politischen, kulturellen und sozialen Bereiche beeinflusste ${ }^{11}$. Eine „,herrenlose Sklaverei“"12 nennt Max Weber ein ökonomisches System, in dem alle Beteiligten ,so streng rational wie eine Maschine ${ }^{\text {"13 }}$ handeln müssen ${ }^{14}$. Der Mensch wurde degradiert „,zu einem Rädchen in dieser Maschine“15. Die Rufe nach mehr Moral in Politik und Wirtschaft wurden einfach ignoriert und waren verpönt. Es kam, wie bei Marx, zu einer Überbetonung der materiellen, ökonomischen Werte. Im Zentrum dieser Weltanschauung steht das Geld/ Kapital und nicht der Mensch und seine Würde ${ }^{16}$. Kardinal Marx schreibt in seinem Buch: „Das Kapital“ folgende kritischen Worte: „Wenn selbst in den Wirtschaftredaktionen deutscher Zeitungen das Lob der Gier gesungen wird, dann ist das ein Beispiel für ein solch perverses Verständnis von Freiheit, gegen das gerade wir Christen entschieden Einspruch erheben müssen. Da müssen wir aufstehen und sagen: Das geht so nicht. Wir lassen nicht zu, dass die Sünde gelobt wird. Und wir lassen auch nicht zu, dass der Sünde

${ }^{10}$, ,...) aus einer solchen Vereinfachung aus wirtschaftlicher Sicht (folgt) eine Engführung, die mehr als problematisch ist, da mit ihr die Gefahr einer Enthumanisierung der Ökonomie und der Reduzierung des Menschen zur bloßen Ressource einhergeht“. N.O. Oermann, Anständig Geld verdienen?, München 2007, S. 20.

11 „Mit dem Kapitalismus totalisiert und verabsolutiert sich ein konkretes politischökonomisches System und unterwirft die Wirklichkeit seinen Gesetzen bzw. Systemzwängen“. P. Schönfelder, Pax-Christi-Kommission Weltwirtschaft (Hg.), Der Gott Kapital. Einführung, Berlin 2006, S. 19.

${ }_{12}$ M. Weber, Wirtschaft und Wissenschaft. Grundriss der verstehenden Soziologie, Tübingen 1972, S. 709.

13 Ebenda.

${ }^{14}$ Gegen diese objektiven Gesetzmäßigkeiten sieht Max Weber keine andere Wahl für den Menschen als sich dieser „Rationalität“ des Systems zu unterwerfen, deshalb spricht er von „herrenloser Sklaverei“. Wo Determinismus (objektiver Prozess, Maschine) herrscht, da gibt es keinen Platz für die Freiheit. Ebenda.

15 Ebenda.

${ }^{16}$ Friedrich August von Hayek fordert eine Haltung der „Demut von den unpersönlichen und anonymen sozialen Prozessen...(..)“. Er sprich vom Wunder des Marktes: „Ich habe absichtlich das Wort Wunder gebraucht, um den Leser aus der Gleichgültigkeit herauszureißen, mit der wir das Wirken dieses Mechanismus als etwas selbstverständliches hinnehmen“. F.A. v. Hayek, Individualismus und wirtschaftliche Ordnung, Salzburg 1976, S. 116; www.fahayek.net/publish/ portrait/HayekPortraet.pdf (26.11.2012). 
die Maske der Freiheit aufgesetzt wird“17. Der Münchner Bischof trifft das uns interessierende Problem auf den Punkt. Erstens stellt er fest, dass in den Medien (wir müssen hinzufügen auch in Bildungsbereichen an den Hochschulen und in der Politik) das Lob der Gier gesungen wird. Es ist, um in biblischen Worten zu sprechen, ein Tanz um das goldene Kalb. Die Freiheit des Marktes darf nicht Zweck an sich selbst bleiben, sie braucht auch staatliche Autorität, um in einem vernünftigen Rahmen ,gezähmt“ zu werden. Wenn die Politik und der Staat aber durch die übermächtige Dominanz der Ökonomie (Globalisierungsproblematik) dazu herabgestuft werden, dass sie lediglich die Freiheit der Kapitalströme garantieren ohne für die sozialen Aspekte eines solchen Modells die Verantwortung zu übernehmen, führt dies dazu, dass die Gewinne privatisiert werden (Gewinne zugunsten einer kleinen einflussreichen Minderheit), während die Verluste auf alle Beteiligten verteilt (sozialisiert) werden ${ }^{18}$. Bis in die frühe Neuzeit galt für die kirchliche Morallehre das Verbot des Zinsnehmens ${ }^{19}$. Das gilt auch für die evangelische Theologie. Luther, Zwingli und Calvin haben Bankgeschäfte strikt abgelehnt ${ }^{20}$. Obwohl heute die katholische Moraltheologie nicht mehr die alten Positionen vertritt, bleibt jedoch zu bedenken, dass die moralischen Beweggründe dafür, dass durch Jahrhunderte ein solches Modell der Profitbeschaffung abgelehnt wurde, nicht unbedeutend sind. Die gegenwärtige Krise ist in ihrem Kern gerade mit der ausufernden und technisch möglichen „Freiheit“ der Banken verbunden. Heutzutage, im Globalisierungszeitalter, wurde die Bereicherung der Wenigen auf Kosten von Vielen technisch in einem bis dahin nie

${ }^{17}$ R. Marx, Das Kapital, München 2008, S. 55. Pax-Christi-Kommission Weltwirtschaft, Der Gott Kapital, Lit. Verlag 2006.

${ }^{18}$ Z. Waleszczuk, Globalizacja solidarnosci, Wroclaw 2007, S. 134-135; Z. Waleszczuk, Między egoizmem a dobrem wspólnym, Kraków 2008, S. 118.

${ }^{19}$ Die Ökonomie hat für Aristoteles keine Eigendynamik. Er sah in der Eigendynamik eine Gefahr für den Bestand der Polis. Aristoteles analysiert die Variationen ökonomischen Austauschs, setzt die Zuständigkeiten für ökonomisches Handeln fest: Kauf, Handel und Geldverleih. Da Geldverleih nichts hervorbringe, außer aus Geld noch mehr Geld, deshalb sprach man anknüpfend an den großen Philosophen im Mittelalter vom Verbot des Zinsnehmens. Aristoteles, Politik, Buch I, 1256b; O. Höffe (Hg.), Aristoteles. Politik, Berlin 2011, S. 43. „Wenn die Logik des Profits die Oberhand gewinnt, werden selbst die Institutionen der Polis von ihm infiziert, und darin wird das der politischen Tugend bestehen“. P. Pellegrin, Hausverwaltung und Sklaverei (I 3-12), in: O. Höffe (Hg.), Aristoteles. Politik, Berlin 2011, S. 43; Die Gesetze im vorindustriellen Zeitalter verboten, seit der Aachener Reichssynode Karl d. Gr. vom 23. 03. 789, das Zinsnehmen auf Grund des bloßen Darlehensvertrages. Vgl. J. Höffner, Christliche Gesellschaftslehre, Kevelaer 2000, S. 237- 238.

${ }^{20}$ R. Marx, Das Kapital, München 2008, S. 85. 
dagewesenen Ausmaß ermöglicht. An dieser Stelle möchten wir nur einige Beispiele erwähnen: „Weltweit leben heute 1 Milliarde Menschen in extremer Armut, sie müssen mit weniger als 1 Dollar am Tag auskommen, was heißt, dass ihr Überleben unmittelbar bedroht ist. Setzt man die absolute Armutsgrenze bei einem Einkommen von weniger als 2 Dollar pro Kopf (ungefähr ein Euro pro Tag) und Tag an, so schätzt man die Zahl derer, die ihr Leben unter dieser Grenze bestreiten müssen, auf über 2,5 Milliarden“21. Dieser Beschreibung des Elends der gegenwärtigen Welt steht die immer steigende Macht und der Reichtum einiger weniger gegenüber: Zwei Prozent der Menschheit verfügt über $40 \%$ des Weltvermögens ${ }^{22}$. Diese himmelschreiende Ungerechtigkeit, bei der augenscheinlich ca. 3\% der Menschen die Hälfte des zur Verfügung stehenden Vermögens in ihrer Hand konzentriert haben, ist eine Folge des dominierenden Systems - der Rechtfertigung der Gier. Dass es so nicht weiter gehen kann und darf, sieht inzwischen jeder vernünftige Mensch ein. Wir müssen jedoch ergänzen, dass die Mächtigen und Einflussreichen dieser Welt diese simple These immer noch nicht einsehen (wollen), denn auch in den größten Krisen, so wie der gegenwärtigen, sind immer noch Profite machbar. „Als der BBC Reporter Greg Palast den Gründer von Donegal, Michael Sheehan, darauf ansprach, ob er bei seinen Geschäften mit der Not der Ärmsten der Armen keine Gewissensbisse habe, antwortete Sheehan ungerührt: Das sind nicht meine Schulden. Ich hatte lediglich die Möglichkeit zu einem

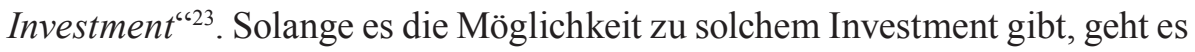
nicht nur um die theoretische Rechtfertigung der Gier, sondern um fehlende praktische Regelwerke, die solche „Freiheit“ der Unternehmen begrenzen und $\mathrm{zu}$ mehr Verantwortung zwingen ${ }^{24}$. Auf die Frage eingehend, warum die Gier gerechtfertigt wurde, können wir im Lichte des oben gesagten eine nüchterne Antwort wagen: Sie wurde nicht im Sinne von Adam Smith zum

21 Ebenda, S. 22.

22 Ebenda.

23 Ebenda, S. 140.

24, ,...) trotz aller Börsencrashs bringen Hedgefonds-Manager noch immer weltweit Firmen und Konzerne durch ,unfreundliche Übernahmen” in Bedrängnis. Ungehindert werden weiter am Devisenmarkt mit Milliardeneinsätzen Währungen manipuliert. Der Poker ist allzu verlockend: Gewinne abschöpfen und sich aus dem Staub machen. Verantwortungsgefühl war gestern. Die Bankerzocker stehen benommen an der Reling und feiern sich ab. Doch wo ist der Kapitän, der ihr Schiff steuert? Es geht nur um den eigenen Wohlstand: mein Auto, meine Villa, meine Südinsel. Hurra, es lebe das eigen Ich. Ist das jetzt nur noch zynisch? Nein, so lautet die nackte Logik unseres Systems“. B. Gorissen, Glaube statt Inflation, Die Tagespost Nr. 142 (27.11.2012), S. 9. 
Wohlstand der Nationen ausgedacht, sondern im Gegenteil zum möglichst schnellen Wohlstand der Macht-, Reichtum- und Informationshabenden missbraucht. „Heute sitzen die Zyniker, die keine Hemmungen haben sich auf Kosten anderer zu bereichern, in aller Regel nicht in Königspalästen, sondern in Büros in New York, London und anderen Metropolen dieser Welt. Anders als die Tyrannen im alten Orient brauchen sie sich bei ihren Beutezügen allerdings nicht auf das eigene Volk zu beschränken, sondern sie können in der ganzen Welt ihr Unwesen treiben" ${ }^{25}$. Gewissenlose Spekulanten können sogar gerade in Krisenzeiten Geschäfte mit den Schulden der Staaten machen. Wir wollen hier die sogenannten „Geierfonds“ (vulture funds) nennen ${ }^{26}$. Wie funktionieren sie? Wie eine Bande Räuber warten die Spekulanten darauf, dass ein Land in die Schuldenkrise gerät (was heutzutage alle Länder der Welt betreffen kann), je schlimmer die Situation des Landes, desto besser, denn die Finanzhaie kaufen die Schulden des konkreten Staates auf und verklagen ihn dann auf Rückzahlung einschließlich Zins und Zinseszinsen. Kardinal Marx erwähnt in diesem Zusammenhang das Beispiel von Peru. Dieser arme Staat hatte 20 Mio. Dollar Schulden im Jahre 1996. Diese Schulden wurden durch einen Spekulanten aus New York, Paul Singer, für 11 Mio. gekauft. Der zynische Milliardär verklagte dann das Land auf Rückzahlung und bekam vor Gericht Recht auf 58 Mio. Dollar. Es lässt sich leicht ausrechnen, dass der „unmoralische“ Unternehmer ohne Mühe 47 Mio. Dollar an Peru verdiente ${ }^{27}$.

\section{Die Gier aus der Sicht der Katholischen Soziallehre}

Wie oben aufgezeigt, ist die Rechtfertigung der Gier die Folge eines verkürzten Menschenbildes. Der Mensch ist in dieser Perspektive ein auf Materialismus und Konsum orientiertes Wesen. Die tieferen, spirituellen, transzendenten Dimensionen werden außer Acht gelassen, als nicht relevant

\footnotetext{
${ }^{25}$ R. Marx, Das Kapital, München 2008, S. 138.

26 „Hier werden Raubzüge auf alles geplant, was schnelles Geld verspricht. Hier handelt man mit Insiderwissen, Devisen, Wertschriften und Waren (auch Grundnahrungsmittel wie Mais und Weizen). Wer hier auf Missernten setzt, baut nicht etwa Getreidespeicher für die Hungernden, sondern verdient sich eine goldene Nase, weil es zu Hungersnöten kommen wird“. B. Gorissen, Glaube statt Inflation, Die Tagespost Nr. 142 (27 XI 2012), S. 9.

${ }^{27}$ R. Marx, Das Kapital, München 2008, S. 139.
} 
für das Ziel des Wirtschaftens, das ebenso verkürzt betrachtet wird ${ }^{28}$. Um das Bibelwort noch einmal zu benutzen: alles dreht sich um das „goldene Kalb“ - Kapital. Wie Papst Johannes Paul II. unermüdlich betonte, geht es um die Frage: mehr Haben oder mehr Sein ${ }^{29}$ ? Durch die Überbetonung des Konsum-, Konkurrenz- und Nützlichkeitsdenkens bleibt der Mensch auf der Strecke.

Der entscheidende Aspekt, aus der Sicht der katholischen Soziallehre, ist das christliche Verständnis vom Menschen auch im Zusammenhang von Markt und Wirtschaft. Der christliche Beitrag zu wirtschaftlichen Fragen verweist auf die philosophische Tatsache, dass alles Handeln der Menschen sittlich ist. Auch im Bereich desMarktessolleskeinewertfreien Räumegeben ${ }^{30}$.An dieserStellekönnen wir auf das sogenannte Bökenförde-Dilemma hinweisen, welches besagt, dass „der freiheitlich säkularisierte Staat von Voraussetzungen lebt, die er selbst nicht garantieren kann ${ }^{\text {“31 }}$. Auch die Ökonomie lebt aus den gleichen Voraussetzungen, Wilhelm Röpke (Vater der sozialen Marktwirtschaft), hatte es folgendermaßen formuliert: ,,diese Kraft erwächst nicht aus dem Markte selber und auch nicht aus dem Spiel der hier sich messenden Interessen, sondern die Menschen müssen sie bereits besitzen, und Familie, Kirche, echte Gemeinschaften und Überlieferung müssen sie damit ausstatten ${ }^{632}$. Um welche Kraft geht es hier? Im Sinne Johannes Pauls II. können wir mit einem Wort formulieren: um die Solidarität (Gemeinsinn

${ }^{28}$ „In der kapitalistischen Gesellschaft wird Geld zum God-term“. J. Hörisch, Kopf oder Zahl. Die Poesie des Geldes, Frankfurt 1996, S. 99.

${ }^{29}$ Z. Waleszczuk, Globalizacja solidarnosci, Wroclaw 2007.

${ }^{30}$ Aristoteles erklärt den Begriff Ökonomie als im Dienst des ganzen Hauses stehend. Das griechische Wort „oikos“ bedeutet Haus und das griechische Verb „nemein“ heißt zuteilen. Aristoteles betont die Rolle der Familie, er spricht von ihrer zentralen Bedeutung für die Gesellschaft. Vgl. P. Pellegrin, Hausverwaltung und Sklaverei (I 3-12), in: O. Höffe (Hg.), Aristoteles. Politik, Berlin 2011, S. 37-56.

${ }^{31}$ „Der freiheitliche, säkularisierte Staat lebt von Voraussetzungen, die er selbst nicht garantieren kann. Das ist das große Wagnis, das er, um der Freiheit willen, eingegangen ist. Als freiheitlicher Staat kann er einerseits nur bestehen, wenn sich die Freiheit, die er seinen Bürgern gewährt, von innen her, aus der moralischen Substanz des einzelnen und der Homogenität der Gesellschaft, reguliert. Anderseits kann er diese inneren Regulierungskräfte nicht von sich aus, das heißt, mit den Mitteln des Rechtszwanges und autoritativen Gebots zu garantieren versuchen, ohne seine Freiheitlichkeit aufzugeben und - auf säkularisierter Ebene - in jenen Totalitätsanspruch zurückzufallen, aus dem er in den konfessionellen Bürgerkriegen herausgeführt hat“. E.W. Bökenförde, Staat, Gesellschaft, Freiheit, Frankfurt 1976, S. 60. A. Sen, Ökonomie für den Menschen. Wege zu Gerechtigkeit und Solidarität in der Marktwirtschaft, Hanser Verlag 2000.

32 W. Röpke, Marktwirtschaft ist nicht genug. Gesammelte Aufsätze, in: Hennecke, Hans Jörg (Hg.), Waltrop 2009, S. 309. „Er muss vielmehr auf die moralischen Kräfte anderer Subjekte zurückgreifen, die diese hervorbringen können“. Ebenda. 
und Verantwortung). Bei Benedikt XVI. geht es hier um die Kraft der Liebe, die alle Bereiche menschlichen Lebens durchdringen soll ${ }^{13}$. Papst Benedikt hat in seiner Sozialenzyklika formuliert: „es geht um die Zivilisierung der Wirtschaft ${ }^{\text {‘34 }}$. Wie Ursula Nothelle-Wildfeuer dazu bemerkt bedeutet das, dass neben dem Markt nicht nur ein Platz für Gewinnmaximierung sein soll, sondern auch für sozial ausgerichtete Produktionsverbände, die nicht nach der Logik des Tausches und Profits handeln, sondern nach der Logik der Gabe. Damit wird z.B. die Leistung der Mütter aufgewertet, die unentgeltlich ,das wertvollste Kapital“ - den Menschen - in seiner Entwicklung durch die Logik der Gabe fördern. Die Wirtschaft muss, wie Kardinal Marx in seinem Buch betont, zurück zu den Menschen finden. Der Bischof von München knüpft an die Ausführungen des Nobelpreisträgers für Ökonomie Armatya Sen an, der in seinem Buch „Ökonomie für den Menschen", das Hauptproblem der gegenwärtigen Krise deutlicher noch in englischer Sprache im Titel folgendermaßen formuliert hat: „Developement

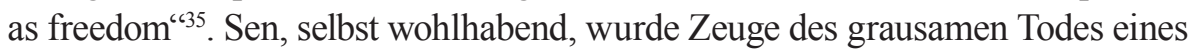
Mannes, der aufgrund der wirtschaftlichen Unfreiheit in Bangladesch starb. Er war ein muslimischer Tagelöhner, der in einem überwiegend hinduistischen Viertel für ein paar Pfennige gezwungen war zu arbeiten. Obwohl seine Frau ihn anflehte so ein gefährliches Viertel zu meiden, war er, ähnlich wie im 19.Jh., aus existentiellen Gründen (seine Familie hungerte) gezwungen sein Leben aufs Spiel zu setzen. In der Einführung zu seinem Buch betont der indische Ökonom, dass diese Kindheitserfahrung der entscheidendste Moment war, der ihn dazu bewegte etwas gegen eine verheerende Hungersnot und Ungerechtigkeit, die er als Kampf für die Freiheit der Menschen betitelte, zu tun. Die Frage der Freiheit bildet das Herz der katholischen Soziallehre. Johannes Paul II. fasst seine Sozialenzykliken zusammen, indem im Zentrum seiner Erwägungen der Mensch und die ihm gebührende Freiheit stehen ${ }^{36}$. „Denn so wie Jesus sich besonders um die Armen und Benachteiligten gekümmert hat, so ist es auch die Aufgabe der Kirche, sich in

${ }^{33}$ Z. Waleszczuk, Prawdziwy rozwój ludzki w epoce globalizacji. Komentarz do encykliki społecznej Benedykta XVI Caritas in veritate, „Homo Dei” 4 (2009), S. 107-112.

${ }^{34}$ Der Papst betonnt in seiner Sozialenzyklika Caritas in veritate dass, (...) das erste $\mathrm{zu}$ schützende und zu nutzende Kapital der Mensch ist, die Person in ihrer Ganzheit”. Benedikt XVI, Caritas in Veritate, Vatican 2009, Nr. 25.

35 A. Sen, Developement as freedom, Oxford 1999; A. Sen, Ökonomie für den Menschen. Wege zu Gerechtigkeit und Solidarität in der Marktwirtschaft, Hanser Verlag 2000; R. Marx, Das Kapital, München 2008, S. 67.

${ }^{36}$ R. Marx, Das Kapital, München 2008, S. 67. 
seiner Nachfolge besonders für die Armen und Benachteiligten einzusetzen und ihre Lebensmöglichkeiten und Freiheitsperspektiven zu verbessern“37.

Das Personalitätsprinzip ist der Dreh- und Angelpunkt der Soziallehre, was besonders deutlich im Denken Karol Wojtylas/Johannes Pauls II. zu sehen ist. Die Würde des Menschen wird definiert in Zusammenhang mit der Befähigung der Person zu moralischen Handlungen. Den zentrale Maßstab jeglichen Handelns hat das Vatikanische Konzil folgendermaßen formuliert: „Wurzelgrund nämlich, Träger und Ziel aller gesellschaftlichen Institutionen ist und muss auch sein die menschliche Person" ${ }^{\text {"38 }}$.

Auch das Wirtschaftsleben ist aus der Sicht der Katholischen Soziallehre diesem Prinzip verpflichtet. Jeder Einzelne und alle Menschen sollen unter menschenwürdigen Entwicklungsbedingungen leben dürfen ${ }^{39}$. Der Fortschritt der Technik und die neuen Möglichkeiten des Wirtschaftens im Globalisierungszeitalter sind nach dem Maßstab zu beurteilen: ist der Mensch ihr primäres Subjekt, dienen sie seiner Entwicklung, oder um auf den kantischen Imperativ zurückzukommen, instrumentalisieren sie nur den Menschen und missachten seine unantastbare Würde? Benedikt schreibt in seiner sozialen Enzyklika, dass „das erste zu schützende und zu nutzende Kapital der Mensch ist, die Person in ihrer Ganzheit ${ }^{640}$. Diese Argumentation von der Würde des Menschen als Person, die zu Freiheit und Liebe berufen ist, ist das Hauptthema des Denkens Karol Wojtylas in Person und Tat. An dieser Stelle wollen wir nicht ausführlicher darüber referieren, sondern lediglich betonen, dass der Papst aus Polen den Primat der Arbeit vor dem Kapital sieht und damit den Menschen ins Zentrum stellt. In seiner ersten Sozialenzyklika Laborem exercens schreibt er, anknüpfend an diese zentrale Frage, über die Würde der Arbeit und das notwendige Verhältnis zwischen Arbeit und Ethik.

Schon der Kirchenvater Augustinus hat unser Hauptthema, die Gier, im Lichte des Evangeliums beschrieben: „Was anders sind also Reiche,

37 Ebenda.

${ }^{38}$ Gaudium et spes, Nr. 25,1; Gaudium et spes Nr. 63, www.vatican.va/archive/hist_councils/ii vatican_council/documents/vat-ii_const_19651207_gaudium-et-spes_ge.html. Vgl. U. NothelleWildfeuer, Markt und Moral? Impulse der katholischen Soziallehre und evangelischen Sozialethik zur Fortentwicklung der Sozialen Marktwirtschaft, www.ordosocialis.de/pdf/Nothelle-Wildfeuer/ P-K-W\%20-\%20Ordo\%20socialis.pdf (23.11.2012).

39 „Ohne rechtschaffene Menschen, ohne Wirtschaftsfachleute und Politiker, die in ihrem Gewissen den Aufruf zum Gemeinwohl nachdrücklich leben, ist die Entwicklung nicht möglich“. Benedikt XVI, CiV 71.

${ }^{40}$ Benedikt XVI, Caritas in veritate, Vatikan 2009, Nr 25. 
wenn ihnen Gerechtigkeit fehlt, als große Räuberbanden? Sind doch auch Räuberbanden nichts anderes als kleine Reiche. Auch das ist eine Schar von Menschen, die unter Befehl eines Anführers steht, sich durch Verabredung zu einer Gemeinschaft zusammenschließt und nach fester Übereinkunft die Beute teilt. Wenn dies üble Gebilde durch Zuzug verkommener Menschen so ins Große wächst, dass Ortschaften besetzt, Niederlassungen gegründet, Städte erobert, Völker unterworfen werden, nimmt es ohne weiteres den Namen Reich an, den ihm offenkundig nicht etwa hingeschwundene Habgier, sondern erlangte Straflosigkeit erwirbt. Treffend und wahrheitsgemäß war darum die Antwort, die einst ein aufgegriffener Seeräuber Alexander dem Großen gab. Denn als der König den Mann fragte, was ihm einfalle, dass er das Meer unsicher mache, erwiderte er mit freimütigem Trotz: $>$ Und was fällt dir ein, dass du das Erdreich unsicher machst? Freilich, weil ich's mit einem kleinen Fahrzeug tue, heiße ich Räuber. Du tust‘s mit einer großen Flotte und heißt Imperator $<" 41$. Der Ausgangspunkt aller sozialethischen Ausführungen ist in dem biblischen Bild von der Erschaffung des Menschen (imago dei) enthalten. Aufphilosophischem Wege gibt Kants Lehre den gleichen Gedanken von der Verpflichtung aller Menschen einander zu achten ${ }^{42}$ aufgrund der ihnen innewohnenden Personenwürde wider. Wie Marx mit Recht betont, ist in diesem Bild „die Gleichheit aller Menschen vor Gott Ausgangspunkt und Maßstab der Leitidee Gerechtigkeit ${ }^{\star 43}$. Im Neuen Testament finden wir ebenfalls eine deutliche Kritik der Habgier „Gebt acht, hütet euch vor jeder Art von Habgier. Denn der Sinn des Lebens besteht nicht darin, dass ein Mensch aufgrund seines großen Vermögens im Überfluss lebt ${ }^{644}$. Auch im

${ }^{41}$ Augustinus, De Civitate Dei IV, 4. Vgl. R. Marx, Das Kapital, München 2008, S. 129.

42 „Ein jeder Mensch“, schreibt Kant in der Metaphysik der Sitten, 2. Teil, § 38, „hat rechtmäßigen Anspruch auf Achtung von seinen Mitmenschen, und wechselseitig ist er dazu auch gegen jeden anderen verbunden“. I. Kant, Metaphysik der Sitten, 2. Teil, A 139, in: W. Weischedel (Hg.), Immanuel Kant. Werke in sechs Bänden, Wissenschaftliche Buchgesellschaft, Darmstadt (1956-1964), Bd. 4, S. 600. „Achtung geht jederzeit auf Personen, niemals auf Sachen“, I. Kant, Kritik der praktischen Vernunft A, S. 35. Siehe: N. Fischer, Sein und Sinn der menschlichen Freiheit, S. 31; N. Fischer, Sein und Sinn der menschlichen Freiheit in der Philosophie Kants, in: Ch. Böttigheimer, N. Fischer, M. Gerwing (Hg.), Sein und Sollen des Menschen, Münster 2009, S. 26; „Im Sinne Kants kann man von einem Postulat sprechen, das besagt: wenn absolute Würde des Menschen sein soll, dann nur, wenn Gott ist und wenn ein Gott des Erbarmens und der Gnade ist“. W. Kasper, Barmherzigkeit. Grundbegriff des Evangeliums - Schlüssel christlichen Lebens, Herder 2012, S. 14.

${ }^{43}$ R. Marx, Das Kapital, München 2008, S. 131.

${ }^{44}$ Lk 12,15. 
184 Volume 3 (2013) Number 2, p. 173-192

Bild des letzten Gerichtes lautet die entscheidende Frage: Was hast du getan? Damit wird die persönliche Verantwortung jedes handelnden Individuums auch im wirtschaftlichen Bereich hervorgehoben: „Und wann haben wir dich krank oder im Gefängnis gesehen und sind zu dir gekommen? Darauf wird der König ihnen antworten, Amen ich sage euch, was ihr für einen meiner geringsten Brüder getan habt, das habt ihr mir getan ${ }^{\text {“45. }}$. In diesem Zusammenhang wird ein gieriges Verhalten, das in unserer heutigen Kultur als Privatangelegenheit abgetan wird, nicht nur als unmoralisch bewertet, sondern im Lichte der Sozialethik als selbstzerstörerisch entlarvt. Johannes Paul II. sprach oft vom Kampf zwischen der Zivilisation der Liebe und der Zivilisation des Todes. Ein falsches Bild vom Menschen und eine ausschließlich auf Materialismus und Nützlichkeit ausgerichtete Ökonomie führen uns alle in die Katastrophe, die wir bereits in den Zeichen der Zeit als Finanzkrise und den daraus resultierenden Folgen von Armut, Unsicherheit und Unruhen bis hin zur Gefährdung des Weltfriedens, beobachten können. „Die wichtigste Ressource ist der Mensch selbst ${ }^{\star 46}$. Jürgen Habermas kritisierte einmal die gegenwärtigen Tendenzen der Wirtschaft, die nicht mehr der Lebenswelt des Menschen dienen, sondern sie kolonialisieren ${ }^{47}$. Das Wirtschaftsleben muss also eingebettet werden in einen umfassend ethischen Horizont ${ }^{48}$.

\section{Was sollen wir tun?}

Gemäß der sozialethischen Methode ist nach der Analyse der Zeichen der Zeit im Lichte des Evangeliums und der Tradition die Frage nach den Auswegen aus der gegenwärtigen Krise relevant. Benedikt XVI., ähnlich wie Johannes Paul II., sieht die Ursachen unserer wirtschaftlichen und politischen Misere in einer falschen Anthropologie verborgen. Die Zivilisierung der Wirtschaft muss nicht nur von oben geschehen, durch neue Ansätze, die die Ökonomie ausdenkt oder die Politik einführt, sondern wie Papst Benedikt deutlich hervorhebt, durch die wachsende Rolle der Zivilgesellschaft. „Die Zivilgesellschaft bringt das Ethos ein, das notwendig ist, um die Wirtschaft

\footnotetext{
${ }^{45}$ Mt 25,33.

${ }^{46}$ Centesimus annus, Nr. 32.

${ }^{47}$ R. Marx, Das Kapital, München 2008, S. 299.

${ }^{48}$ Siehe: Benedikt XVI, Caritas in veritate, Vatican 2009.
} 
zu ihrem eigentlichen Ziel zu führen, um ganzheitliche Entwicklung gelingen zu lassen ${ }^{، 49}$.

Die Kirche liefert keine technischen Lösungen und präzisen Reformkonzepte ${ }^{50}$. Einerseits soll sie die berechtigte Autonomie von Wirtschaft und Politik nicht so weit überschreiten, andererseits jedoch wird aus den oben gezeigten Tendenzen und Gefahren für das Wohl der Menschheit und vor allem der Schwachen und Benachteiligten deutlich, dass sie nicht schweigen darf. Die Vorschläge, die die Sozialethik formuliert, betreffen nicht nur die Kirche als Gemeinschaft der Glaubenden, sondern die ganze Menschheitsfamilie. Gerade deshalb, weil sie sich auch in Wirtschafts- und Politikfragen einmischen muss, wird sie heutzutage so heftig angegriffen durch jene Machthaber, die unsere immer mehr globale Wirtschaft, Politik und Medienwelt dominieren. Die skrupellosen, zynischen und gierigen „Unternehmer“ die weiterhin ohne Ethos auskommen, attackieren deshalb ohne Pardon die katholische Kirche und die Christen weltweit so heftig, weil ihre Stimme eine der letzten hörbaren Gewissensrufe ist.

Bei der Aufgabe, der Tugend im wirtschaftlichen und politischen Bereich mehr Geltung zu verleihen, darf und kann die Soziallehre sich keinen Rückzug leisten. Es geht, wie Paul VI. (PP), Johannes Paul II. (SRS) und Benedikt XVI. $(\mathrm{CV})$ in Bezug auf unsere immer stärker zusammenwachsende Welt sagen, um die wahre Entwicklung des Menschen, was mit dem Heilsanspruch der Kirche zusammenhängt. Alles was den Menschen an seiner Bestimmung hindert, seine Freiheiten raubt, ihn zum Sklaven der ökonomischen, gierigen Machtspiele macht, muss aus der Sicht der Kirche nicht nur angeprangert werden, sondern auch durch die gemeinsame Anstrengung aller Menschen guten Willens, die sich der Ernsthaftigkeit unserer Lage bewusst sind, geändert und auf eine humanere Weltordnung (Wirtschaftsordnung) hingearbeitet werden.

Christliche Sozialethik liefert zwar keine technischen Lösungen, gleichzeitig jedoch, ist sie im gesellschaftlichen Leben, wie Lothar Roos feststellt, wirksam gewesen $^{51}$. Unsere Konsumgesellschaft, die durch die Rechtfertigung der Gier in

${ }^{49}$ U. Nothelle-Wildfeuer, Markt und Moral? Impulse der katholischen Soziallehre und evangelischen Sozialethik zur Fortentwicklung der Sozialen Marktwirtschaft, www.ordosocialis. de/pdf/Nothelle-Wildfeuer/P-K-W\%20-\%20Ordo\%20socialis.pdf. S. 12 (23.11.2012).

${ }^{50}$ Z. Waleszczuk, Globalizacja solidarności, Wrocław 2007, S. 398-399.

${ }^{51}$ L. Roos, Die Katholische Soziallehre und die Reform des Sozialstaates, www.bku.de/html/ img/pool/disk29kathsoz.pdf (23.11.2012); L. Roos, Rheinischer Merkur vom 9.07.2009; H. Kues, Wegweiser in der Krise? Die Enzyklika caritas in veritate gibt der Soziallehre neuen Schwung, 
die Krise geraten ist, verlangt nach neuen, internationalen Finanzmarktregelungen, was mit der Notwendigkeit moralischer Fundamente zu tun hat. Gerade weil es in den modernen ökonomischen Theorien zum Ausschluss der Ethik gekommen ist und die entfesselten globalen Märkte ihr ganzes Vertrauen lediglich auf die unsichtbare Hand des Marktes und die Technik der Profitmaximierung gesetzt haben, sehen wir allmählich (und sollten wie Papst Benedikt zum einzig vernünftigen Schluss kommen): „,es gibt überhaupt kein wirtschaftliches Handeln ohne Moral ${ }^{652}$. Alle Reformkonzepte, die sich lediglich auf Veränderungen auf dem Finanzmarkt oder der Kreditpolitik konzentrieren, greifen, aus der Sicht der Kirche, zu kurz, wenn siekeine sozialethischen Forderungen im öffentlichen Leben (sowie in Wirtschaft und Politik) abverlangen. Der ehemalige Bundespräsident Horst Köhler, der renommierte Ökonom und ehemalige Direktor des IWF, sagte in seiner Berliner Rede im April 2009 sehr treffend, dass ,den Ratingagenturen und Banken bei ihren gierigen Rennen nach den schnellstmöglichen und maximalen Renditen die rechte Haltung abhanden gekommen sei: So etwas tut man nicht ${ }^{453}$. Die Frage nach ethischen Prinzipien auch im Wirtschaftsund Finanzwesen ist eine zentrale Frage der Sozialethik, gerade deshalb, weil wir in einer Zeit leben, in der die Grundbegriffe unserer Kultur in Frage gestellt werden. Papst Benedikt stellte bei seiner Heimatvisite im Jahre 2011 im deutschen Bundestag ${ }^{54}$ eine relevante Frage, die er aus dem Alten Testament den heutigen Bundestagsabgeordneten in Erinnerung rief. Am Beispiel von König Salomo fragt er, was das Wichtigste für einen König (Politiker, Machthaber auch in wirtschaftlichen Belangen) ist, ist es Macht, oder Reichtum, Vernichtung der Feinde usw... .? Salomo bittet um die Fähigkeit das Gute vom Bösen unterscheiden zu können, um richtige Entscheidungen treffen zu können. Was lernen wir aus dieser Geschichte? Im Sinne Benedikts sehen wir, dass die heutige Elite gerade diese entscheidende Frage ausblendet und sie als irrelevant für wirtschaftliche und politische Handlungen betrachtet. In Theorie und Praxis des wirtschaftlichen

S. 5, www.kas.de/wf/doc/kas_18249-544-1-30.pdf?100105164141 (23.11.2012). „Unbestreitbar ist (...) das Verdienst der katholischen Soziallehre: Sie vermochte, ein geschlossenes, theologisch anspruchsvoll begründetes Modell zu entwickeln, dem es unter anderem gelungen ist, das geistige Fundament der Sozialen Marktwirtschaft der Bundesrepublik Deutschland über Jahrzehnte zu prägen“. N.O. Oermann, Anständig Geld verdienen?, München 2007, S. 34.

${ }^{52} \mathrm{H}$. Kues, Wegweiser in der Krise? Die Enzyklika caritas in veritate gibt der Soziallehre neuen Schwung, S. 7, www.kas.de/wf/doc/kas_18249-544-1-30.pdf?100105164141 (23.11.2012).

${ }^{53}$ Ebenda, S. 8.

${ }^{54}$ Rede Papst Benedikts XVI. im Deutschen Bundestag am 22. September 2011, www. bundestag.de/kulturundgeschichte/geschichte/gastredner/benedict/rede.html. 
Lebens herrscht sogar eine totale Vermischung der Werte, d.h. was in den letzten 3000 Jahren (siehe Prophet Amos) als verwerflich bewertet wurde, nämlich die Gier, wird heute gerechtfertigt und positiv hoch gepriesen. Wenn man keine Orientierungsnormen im Finanzwesen und Unternehmertum will, so heißt das, dass solche Strukturen immer inhumaner werden. Johannes Paul II. bemerkte in CA kritisch, dass die Demokratie ohne Werte zum Totalitarismus wird ${ }^{55}$. Aus der Geschichte des Herrschers Salomo lernen wir, dass er von Gott diese wesentliche Gabe erbittet. Damit ist aus der Sicht Benedikts, die wichtige Relation des Menschen zu seinem Schöpfer zu betonen: ohne Gott verliert der Mensch die letztendliche Begründung seiner moralischen Handlungen ${ }^{56}$. Die Krise unserer Kultur hängt auch damit zusammen, dass der westliche Mensch auf seine eigene absolute Autonomie vertraut und versucht die Welt in allen Bereichen ohne Relation zu Gott zu gestalten. Die gegenwärtige Verunsicherung als Folge einer Wirtschaft ohne Moral verlangt nach Konsequenzen, die aber bis heute nicht gezogen wurden. Die Verursacher der Weltkrise, Manager aus der Wall Street und anderen amerikanischen Kredit- und Finanzinstrumenten, wurden mit Milliarden von Dollars bestens entlohnt, auch im Falle der Insolvenz, was einem elementaren Empfinden nach Gerechtigkeit widerspricht. Anstatt die hauptverantwortlichen Banken, die durch ihr System Manager und Ratingagenturen unter Druck gesetzt haben, durch gieriges und unverantwortliches Handeln zu schnellstmöglichen Profiten zu gelangen, zu bestrafen, werden ihre Schulden, die sie selbst verursacht haben, sozialisiert, d.h. vom Staat übernommen und auf alle Bürger umgewälzt. Ist das gerecht, dass die Profite privatisiert werden und die Schulden sozialisiert? Gibt es eine Autorität, die sich dieser himmelschreienden Ungerechtigkeit widersetzen kann? Gerade weil die katholische Kirche als erster Globalplayer (als letzter wirksamer Anwalt der Armen) auch im Wirtschaftsleben sich für ein altruistisches Ethos stark macht, dürfen wir uns nicht wundern, dass sie durch

55 „Eine wahre Demokratie ist nur in einem Rechtsstaat und auf Grundlage einer richtigen Auffassung vom Menschen möglich“, Johannes Paul II, Centesimus annus 46; ,Wenn nämlich keine letzte Wahrheit anerkannt wird, die das politische Handeln leitet und ihm Orientierung gibt, können Ideen und Überzeugungen nur allzu leicht für Machtzwecke mißbraucht werden. Eine Demokratie ohne Werte verwandelt sich [...] leicht in einen offenen oder hinterhältigen Totalitarismus“, Johannes Paul II, Centesimus annus 46.

${ }^{56}$ R. Spaemann sagt dazu: Das Naturrecht könne, ,uns lehren, was richtig ist, aber es kann uns nicht verpflichten, es zu tun“. ,Entweder steht hinter der befehlenden Autorität die Autorität Gottes oder es gibt überhaupt keine Autorität“. Siehe: S. Rehder, Das Naturrecht in der Denker-Werkstatt, „Die Tagespost“" (6 XI 2012), Nr. 133, S. 3. 
die mainstream-Medien, die in der Hand der gegenwärtigen Herrscher der Welt liegen, so heftig angegriffen wird.

Horst Köhler formulierte in diesem Sinne seine Kritik an einer ausschließlich auf Gewinn ausgerichteten Wirtschaftspolitik: „die große Chance der Krise besteht darin, dass jetzt alle erkennen können: keiner kann mehr dauerhaft Vorteil nur für sich schaffen. Die Menschheit sitzt in einem Boot. Und die in einem Boot sitzen sollen sich helfen. Eigennutz im 21. Jahrhundert heißt: sich umeinander kümmern“57. Vielleicht gerade deshalb, weil Horst Köhler so mutige Worte fand und öffentlich verriet, dass die deutschen Soldaten in Afghanistan aus wirtschaftlichen Gründen sind, was mit unserem Thema - die Gier nach mehr Haben - zu tun hat, musste er sein Bundespräsidentenamt auf den Wunsch unserer „pluralistischen“ Medien räumen.

Obwohl das Ziel der christlichen Soziallehre nicht in präzisen technischen Reformkonzepten besteht, können wir, aufgrund der Auftragsverpflichtung durch das Evangelium uns um das Menschenwohl (der Menschheitsfamilie) zu bemühen, auf die Frage nach dem „was sollen wir tun?“, einige relevante Postulate formulieren. Zusammenfassend lässt sich die gegenwärtige Krise als ein Spannungsverhältnis zwischen mehr Haben oder Sein, der Priorität des Kapitals vor der Arbeit, den Strukturen der Sünde vor der Zivilisation der Liebe (vgl. Johannes Paul II.) definieren. Wenn meine Gier nur durch die Macht begrenzt wird (der Stärkere gewinnt), wenn diese „Tugend“ alle anderen zivilgesellschaftlichen Tugenden erstickt, befinden wir uns auf dem direkten Weg zur Selbstzerstörung. Was können wir tun? Aus dem oben Gesagten lässt sich leicht feststellen, dass die heutige Rechtfertigung der Gier mit der katholischen Soziallehre nicht vereinbar ist, der Verbannung der Ethik aus dem öffentlichen Leben muss ein Ende gesetzt werden. Wir können deshalb folgende Postulate, die zu mehr Gemeinwohl führen, formulieren:

- Ethik als Fundament einer gerechten Ordnung (Gott als Garant der Wahrheit und Autorität der Moral - ohne Gott verliert der Mensch die letzte Begründung, warum er moralisch handeln soll, was zu Orientierungslosigkeit und Relativismus führt)

- Primat der Person (Familie, Menschheitsfamilie)

${ }^{57}$ H. Kues, Wegweiser in der Krise? Die Enzyklika caritas in veritate gibt der Soziallehre neuen Schwung, S. 7, www.kas.de/wf/doc/kas_18249-544-1-30.pdf?100105164141, S. 8 (23.11.2012). 
- Bildung - der gemeinsamen Handlung eine Orientierung geben (an den Universitäten gehört die Sozialethik leider nicht zu den Kernfächern der Theologie; ihre Bedeutung wird unterschätzt!)

- Solidaritätsbewusstsein reifen lassen, d.h. praktisch nicht weiterhin die „Tugend“ der Gier, sondern die wahren Tugenden (Gerechtigkeit, Mäßigung, Mildtätigkeit,...) wiederentdecken und fördern

- Primat der Politik (das Finanzwesen soll durch entsprechende Regeln auf globaler Ebene zu mehr Verantwortung gezwungen werden)

- Dem Staat zu mehr Demokratie verhelfen (Subsidiarität fördern)

- Kontrolle der Banken (z.B. Geierfonds und andere moralisch zweifelhafte Praktiken der Gewinnmaximierung sind zu unterbinden)

- Spekulanten und Betrüger werden zur Verantwortung gezogen (bis heute genießen sie trotz weniger Ausnahmen große Privilegien und werden überdimensional honoriert)

- Internationalisierung des zivilgesellschaftlichen Engagements (im Sinne des Subsidiaritätsprinzips Bildung von Staatsgrenzen überschreitenden Institutionen, Organisationen und Vereine, die die Interessen der Benachteiligen wirksamer verteidigen können)

- „kulturellen Fortschritt“ neu definieren - gegen den Strom der sich auflösenden westlichen Kultur schwimmen (Pädagogik des Vertrauens und der Verantwortung)

- Das Recht auf Religionsfreiheit verteidigen und schützen

- Aufwertung der Logik des Schenkens (z.B. eine Mutter, die sich entscheidet anstatt Karriere zu machen fünf Kinder zu erziehen, wird nicht entsprechend geachtet und für ihre Leistung honoriert, sondern eher steuerlich bestraft)

- Relativierung der ausschließlichen Ausrichtung auf Gewinn (z.B. im hohen Alter werden bestimmte medizinische Eingriffe als ,,nicht mehr lohnend" bewertet, was an eine Verletzung der Menschenwürde grenzt)

- Stärkung und Internationalisierung der Gewerkschaften

- Humanisierung der Arbeitswelt

- Pluralisierung der Medien (in Wirklichkeit sehen wir die umgekehrten Tendenzen, (z.B. in Polen) die sich als Monopolisierung der Macht für die dominierenden Konzerne bezeichnen lassen $)^{58}$

${ }^{58}$ B. Bubula, Podatek od prawdy, www.naszdziennik.pl/mysl/16231, podatek-od-prawdy.html. (03.12.2012). 
- Förderung der Weisheit (Liebe und Wissen) in der Politik, Wirtschaft und im öffentlichen Leben - in Wirklichkeit fehlt es heute an „richtungsweisenden Systemen“59.

Auch wenn die oben beispielhaft aufgezählten Postulate, die für manche lediglich wie ein Appell oder eine Utopie klingen mögen, keinen Anspruch auf Ausschließlichkeit und Vollständigkeit erheben und das Thema nicht ausschöpfen, sind sie aus der Perspektive der Soziallehre (vor allem anknüpfend an die Lehre von Johannes Paul II. und Benedikt XVI.) als unverzichtbare Voraussetzungen zur wahren Entwicklung des Menschen zu betrachten. Im Sinne der christlichen Sozialethik sind der Ursprung, die Gegenwart und das Ziel aller öffentlichen (auch wirtschaftlichen) Tätigkeiten der Menschheitsfamilie die menschliche Person (in individueller und sozialer Dimension), der wir - im Sinne der oben aufzählten Postulate - zu einer effektiven und wirksamen Antwort auf die Herausforderungen des Materialismus verhelfen sollen.

\section{Fazit}

Zusammenfassend lässt sich sagen, dass die gegenwärtige Krise, trotz aller bedrohenden Herausforderungen auch als Chance zu bewerten ist. Die Fokussierung auf die institutionellen Reformen, der Glaube an die Maßnahmen, die ausschließlich soziologische Schwerpunkte betonen und zu sehr auf die Veränderung der Strukturen (Schulbildung, Pädagogik-, Politik-, Wirtschaft-, Sozialwesen) ihr Vertrauen setzen, haben sich als nicht realistisch und erfolgversprechend entpuppt. Erst wenn der Faktor Mensch als Person in seiner unantastbaren Würde wieder seine ihm gebührende Bedeutung auch im Wirtschaftsleben gewinnt, wenn wir danach streben im gesellschaftlichen Leben das Gute vom Bösen zu unterscheiden und die Welt nicht mehr nach dem Prinzip, der Stärkere und Gewissenlose darf mehr Rechte haben, behandeln, sondern nach mehr Gerechtigkeit, die aus der Wahrheit in Liebe erwächst trachten, erst dann ist es möglich da u erh a fte Vorteile für alle Beteiligten (Menschheitsfamilie) wirksam zu erreichen. Andernfalls steuern wir auf eine unvermeidliche Konfliktzunahme hin, die mit dem Begriff Unfrieden und Krieg in globaler Dimension zusammenhängt. Es ist deshalb eine Pflicht nicht nur für das Lehramt der Kirche, nicht nur für jeden Christen, sondern für jeden Menschen guten Willens sich in sozialethischen Fragen zu engagieren. Wenn es

\footnotetext{
${ }^{59}$ „Das Wissen ist niemals allein das Werk der Intelligenz“, Benedikt XVI CiV 30.
} 
um die Frage des Menschen in seiner tiefsten Dimension, um die Ökologie der Person geht, kann die Kirche nicht schweigen, denn ihr Auftrag und Weg ist der Mensch. Die Prinzipien wie Transparenz, Ehrlichkeit, Vertrauenswürdigkeit stehen im Gegensatz zur kaltherzigen Gier. Alle Arten von „Messianismen“ ${ }^{“ 60}$ (z.B. die Rechtfertigung der Gier, und der Glaube an die freie Hand des Marktes) die den Einzelnen, sowie einigen Sektoren des gesellschaftlichen Lebens moralfreie Bereiche garantieren wollen und einen verantwortungsfreien Handlungsspielraum in die Hand geben, unterhöhlen die Grundlagen unserer auf dem Christentum basierenden Kultur. Erst wenn wir uns wie Salomo an Gott wenden und ihn um Hilfe bei der Urteilsbildung und um die Fähigkeit das Gute vom Bösen, das Gerechte vom Ungerechten unterscheiden zu können bitten, finden wir die stabile Grundlage, die uns aus Verzweiflung und Orientierungslosigkeit in eine humanere Zivilisation der Menschlichkeit und Solidarität führen kann.

\section{Bibliography}

Augustinus, De Civitate Dei, (Hg.) Horn Ch., Berlin 1997; www.thelatinlibrary.com/august. html.

Benedikt XVI, Caritas in veritate, Vatican 2009.

Bökenförde E.W., Staat, Gesellschaft, Freiheit, Frankfurt 1976.

Diez A., Der homo oeconomicus in der Perspektive theologischer Wirtschaftsethik, Heidelberg 2004.

Fischer N., Sein und Sinn der menschlichen Freiheit in der Philosophie Kants, in: Böttigheimer Ch., Fischer N., Gerwing M. (Hg.), Sein und Sollen des Menschen, Münster 2009.

Gorissen B., Glaube statt Inflation, „Die Tagespost“ Nr. 142 (27 XI 2012), S. 9.

Hayek F.A. v., Individualismus und wirtschaftliche Ordnung, Salzburg 1976.

www.naszdziennik.pl/mysl/16231,podatek-od-prawdy.html (03.12.2012).

www.unilu.ch/files/die-zeichen-der-zeit-erkennen.pdf (30.08.2012).

www.vatican.va/archive/hist_councils/ii_vatican_council/documents/vat-ii_const_196 51207_gaudium-et-spes_ge.html.

www.fahayek.net/publish/portrait/HayekPortraet.pdf (26.11.2012).

Höffe O. (Hg.), Aristoteles. Politik, Berlin 2011.

Höffner J., Christliche Gesellschaftslehre, Kevelaer 2000.

Hörisch J., Kopf oder Zahl. Die Poesie des Geldes, Frankfurt 1996.

Johannes Paul II, Centesimus annus, www.vatican.va/holy_father/john_paul_ii/encyclicals/ documents/hf_jp-ii_enc_01051991_centesimus-annus_ge.html (05.12.2012).

\footnotetext{
${ }^{60}$ Pax Christi-Kommission Weltwirtschaft (Hg.), Der Gott Kapital, Berlin 2006.
} 
Kant I., Metaphysik der Sitten, 2. Teil, A 139, in: Weischedel W. (Hg.), Immanuel Kant. Werke in sechs Bänden, Wissenschaftliche Buchgesellschaft, Darmstadt (1956-1964), Bd. 4.

Kasper W., Barmherzigkeit. Grundbegriff des Evangeliums - Schlüssel christlichen Lebens, Herder 2012.

Kues H., Wegweiser in der Krise? Die Enzyklika caritas in veritate gibt der Soziallehre neuen Schwung, www.kas.de/wf/doc/kas_18249-544-1-30.pdf?100105164141 (23.11.2012).

Marx R., Das Kapital, München 2008.

Nothelle-Wildfeuer U., Markt und Moral? Impulse der katholischen Soziallehre und evangelischen Sozialethik zur Fortentwicklung der Sozialen Marktwirtschaft, www. ordosocialis.de/pdf/Nothelle-Wildfeuer/P-K-W\%20-\%20Ordo\%20socialis.pdf (23.11.2012).

Oermann N.O., Anständig Geld verdienen?, München 2007.

Pax Christi-Kommission Weltwirtschaft (Hg.), Der Gott Kapital, Berlin 2006.

Pellegrin P., Hausverwaltung und Sklaverei (I 3-12), in: O. Höffe (Hg.), Aristoteles. Politik, Berlin 2011, S. 37-56.

Rede Papst Benedikts XVI. im Deutschen Bundestag am 22. September 2011, www.bundestag. de/kulturundgeschichte/geschichte/gastredner/benedict/rede.html (23.11.2012).

Rehder S., Das Naturrecht in der Denker-Werkstatt, „Die Tagespost“ (06.11.2012).

Röpke W., Marktwirtschaft ist nicht genug. Gesammelte Aufsätze, in: Hennecke, Hans Jörg (Hg.) Waltrop 2009.

Roos L., Die Katholische Soziallehre und die Reform des Sozialstaates, www.bku.de/html/ img/pool/disk29kathsoz.pdf (23.11.2012).

Roos L., Rheinischer Merkur vom 9 VII 2009.

Sen A., Developement as freedom, Oxford 1999.

Sen A., Ökonomie für den Menschen. Wege zu Gerechtigkeit und Solidarität in der Marktwirtschaft, Hanser Verlag 2000.

Schönfelder P., Pax-Christi-Kommission Weltwirtschaft (Hg.), Der Gott Kapital. Einführung, Berlin 2006.

Smith A., Der Wohlstand der Nationen, München 2001.

Weber M., Wirtschaft und Wissenschaft. Grundriss der verstehenden Soziologie, Tübingen 1972.

Waleszczuk Z., Między egoizmem a dobrem wspólnym, Kraków 2008.

Waleszczuk Z., Kłamstwo „,homo oeconomicus” a kryzys kultury, „Życie Konsekrowane” 3 (2010), S. 52-61.

Waleszczuk Z., Globalizacja solidarnosci, Wroclaw 2007.

Waleszczuk Z., Prawdziwy rozwój ludzki w epoce globalizacji. Komentarz do encykliki społecznej Benedykta XVI Caritas in veritate, „Homo Dei” 4 (2009), S. 107-112. 